\title{
Inventer une géométrie pour l'école primaire au XIXe siècle
}

\section{Renaud D'Enfert}

\section{(2) OpenEdition}

1 Journals

Édition électronique

URL : http://journals.openedition.org/trema/1536

DOI : 10.4000/trema.1536

ISSN : 2107-0997

Éditeur

Faculté d'Éducation de l'université de Montpellier

\section{Édition imprimée}

Date de publication : 1 septembre 2003

Pagination : 41-49

ISSN : 1167-315X

\section{Référence électronique}

Renaud D'Enfert, «Inventer une géométrie pour l'école primaire au XIXe siècle », Tréma [En ligne], 22 | 2003, mis en ligne le 13 octobre 2010, consulté le 30 avril 2019. URL : http:// journals.openedition.org/trema/1536 ; DOI : 10.4000/trema.1536

Ce document a été généré automatiquement le 30 avril 2019.

Trema 


\title{
Inventer une géométrie pour l'école primaire au XIXe siècle
}

\author{
Renaud D'Enfert
}

1 En recommandant aux maîtres d'associer les enseignements de géométrie, de dessin et de travail manuel, les programmes et instructions ministérielles de 1909 pour les écoles primaires supérieures, et de 1923 pour les écoles primaires élémentaires, prônent une formule qui va perdurer jusqu'aux années 1960, et dont on retrouve, aujourd'hui encore, des traces dans l'enseignement mathématique dispensé à l'école élémentaire ou au collège : une géométrie essentiellement concrète, s'appuyant largement sur l'activité des élèves par des travaux de dessin ou des réalisations spatiales.

2 Cette coopération disciplinaire, et plus particulièrement celle de la géométrie et du dessin, trouve ses racines dans la façon dont s'est constitué l'enseignement primaire de la géométrie au XIX ${ }^{\mathrm{e}}$ siècle. La géométrie (et non la géométrie pratique, la différence est importante, on le verra) est officiellement introduite dans l'enseignement primaire sous la monarchie de Juillet : la loi Guizot du 28 mars 1833 inscrit "les éléments de la géométrie » au programme de l'enseignement primaire supérieur (de garçons) créé afin d'offrir « à une partie nombreuse de la population une culture un peu plus relevée que celle que lui donnait jusqu'ici l'instruction primaire $"^{1}$. Cette mesure, prise dans un contexte politique favorable à l'instruction populaire, est importante à plus d'un titre : non seulement elle diversifie un enseignement mathématique primaire le plus souvent limité à la seule arithmétique, mais elle ajoute à l'instruction primaire (l'école du peuple) une discipline traditionnellement réservée à l'enseignement secondaire (l'école des notables) ${ }^{2}$. C'est dans cette tension entre, d'une part, la volonté d'élever et de diversifier les études primaires, et, d'autre part, l'exigence sociale et politique de différenciation, en termes de méthodes et de finalités, des études primaires et des études secondaires, que va s'élaborer la géométrie primaire au cours $\mathrm{du} \mathrm{XIX}^{\mathrm{e}}$ siècle. Nous voudrions retracer ici cette élaboration disciplinaire et en montrer les différents enjeux, pédagogiques, épistémologiques, voire idéologiques. 


\section{Mesurer, dessiner}

3 En portant «les éléments de la géométrie et ses applications usuelles, spécialement le dessin linéaire et l'arpentage " - c'est la formulation complète - au nombre des matières de l'enseignement primaire supérieur, la loi Guizot de 1833 inscrit l'enseignement primaire de la géométrie dans une double tradition : celle du mesurage et de la géométrie pratique d'une part, et celle du dessin d'autre part. La première, liée à la mesure des surfaces et des volumes, est la plus ancienne. Déjà, sous l'Ancien Régime, certains maitres d'école se livraient à des opérations d'arpentage (pour évaluer la superficie des terrains) ou en donnaient des leçons. Sous la Révolution, les grands projets d'instruction publique visent à institutionnaliser de telles pratiques. Ainsi, en 1791, le plan de Mirabeau prévoit que les maîtres d'école enseigneront "à calculer et même, s'il est possible, à lever des plans et à arpenter ». L'année suivante, Condorcet propose que l'on enseigne, dans les écoles primaires, "des méthodes simples de mesurer exactement un terrain, de toiser un édifice ». Mais la législation issue de la période révolutionnaire maintient finalement l'enseignement primaire dans le cercle étroit du " lire, écrire, compter ». Il faut attendre 1816 pour que les instituteurs titulaires du brevet du premier degré (c'est-à-dire les plus qualifiés, qui exercent généralement dans les villes importantes) voient l'arpentage entrer officiellement dans leurs attributions.

4 La seconde tradition, qui concerne le dessin, est plus récente, du moins dans ses formes codifiées et scolarisées. Elle remonte aux premières années de la Restauration lorsqu'un enseignement de "dessin linéaire » est introduit dans les écoles mutuelles. Le dessin linéaire - on dit parfois «dessin géométrique » - est une nouvelle méthode de dessin conçue par le mathématicien Louis-Benjamin Francoeur afin de mettre cet enseignement à la portée du peuple 3 . Publiée en 1819 , celle-ci connaît un large succès, d'abord dans les écoles mutuelles, ensuite dans l'ensemble de l'instruction primaire. Elle vise à exercer l'œil et la main, à développer la précision et le sens de l'observation, mais aussi à initier au «bon goût» par des modèles inspirés de l'art antique. Concrètement, les élèves dessinent à main levée, c'est-à-dire sans règle ni compas, des droites, des angles et des figures rectilignes, puis des lignes courbes et des figures curvilignes, qu'ils combinent ensuite dans des figures géométriques plus complexes ou des dessins d'ornement et d'architecture. La progression suit donc un ordre croissant de difficulté des tracés, et non celui des théorèmes de la géométrie classique. Dès la fin des années 1820 , les exercices de dessin à main levée sont complétés par des tracés à la règle et au compas, qui donnent "plus de sûreté et de correction » : les élèves tracent une seconde fois, avec les instruments, l'ensemble des figures qu'ils ont auparavant dessinées à main levée.

5 À la fin des années 1820, géométrie pratique et dessin linéaire tendent parfois à se confondre. Car cette "géométrie populaire» qu'est le dessin linéaire permet de familiariser les élèves avec le calcul des surfaces et des volumes, mais aussi avec le système métrique, encore peu répandu. Paraît ainsi, en 1827, un manuel intitulé de façon significative Dessin linéaire et géométrie pratique ${ }^{4}$. De même, la troisième édition du manuel de Francoeur, Dessin linéaire et arpentage (1832) comprend une nouvelle section consacrée à l'arpentage et au lever des plans, "sujet d'une grande utilité [...] et qui se rattache d'une manière si naturelle au tracé géométrique $»^{5}$.

6 L'enseignement conjoint, au niveau primaire, des enseignements de géométrie pratique et de dessin linéaire trouve donc sa traduction législative avec la loi Guizot de 1833. Mais 
alors que le projet du ministre mettait au premier plan le dessin linéaire et l'arpentage, et, d'une façon plus générale, les "applications de la géométrie pratique ", le texte définitif va au-delà de ses intentions initiales: le législateur prévoit en effet que les élèves des écoles primaires supérieures se verront enseigner «les éléments de la géométrie », dont le dessin linéaire et l'arpentage ne sont que des « applications usuelles». De fait, la loi Guizot établit, dans l'instruction primaire supérieure, un enseignement de géométrie qui fait office de cadre théorique, pour les activités de dessin comme pour celles relatives au mesurage.

7 Cette mise au premier plan de la géométrie, discipline foncièrement spéculative, n'est pas sans conséquences. Ainsi, l'administration ministérielle doit veiller à ce que l'enseignement primaire de la géométrie ne se calque pas sur l'enseignement secondaire, plus théorique et plus abstrait. Car, rappelons-le, au XIX siècle, l'enseignement primaire, élémentaire ou supérieur, qui offre des études courtes aux classes "inférieures" ou «intermédiaires » de la société, doit revêtir un caractère essentiellement pratique, en prise sur la vie quotidienne ou professionnelle ; l'enseignement secondaire, en revanche, propose généralement, à l'intention des classes aisées, un enseignement de culture conduisant au baccalauréat, où domine l'étude des langues anciennes. La différenciation joue moins sur les contenus que sur les méthodes. Un principal de collège, pourtant favorable à l'ouverture d'une école primaire supérieure au sein de son établissement, déclare ainsi en 1837: "On ne doit pas perdre de vue que l'enseignement primaire, même supérieur, differe essentiellement de l'enseignement secondaire, moins par les objets des études que par la méthode. Au collège, la théorie domine la pratique [...]. À l'école au contraire, c'est la pratique qui doit dominer $»^{7}$. Aussi les finalités du dessin linéaire s'en trouvent-elles notablement modifiées. Son enseignement doit préparer au cours de géométrie auquel il est étroitement lié, mais sert en même temps à appliquer les notions théoriques étudiées. Ainsi, après 1833, certains manuels de géométrie offrent des applications pratiques en fin de chapitre telles que tracés de moulures, dallages ou parquetages ${ }^{8}$. Dans les écoles primaires supérieures, les exercices de lever de plans, qui conjuguent dessin et géométrie pratique, constituent bien souvent le couronnement du cours de géométrie. S'esquisse ainsi une géométrie ancrée dans le concret, privilégiant la description et le tracé des figures plutôt que les démonstrations. Le raisonnement, parce qu'il permet de fixer dans la mémoire les notions étudiées, n'est toutefois pas totalement écarté.

8 Un tel enseignement de géométrie concerne a priori le « haut » enseignement primaire : écoles primaires supérieures, mais aussi écoles normales primaires, qui assurent la formation des maîtres. En revanche, il ne vise pas ouvertement l'enseignement primaire élémentaire, et moins encore l'enseignement féminin. Au niveau élémentaire en effet, l'enseignement mathématique n'embrasse, selon la loi Guizot, que les "éléments du calcul » et le "système légal des poids et mesures». Mais le législateur a prévu d'accorder une certaine latitude aux établissements "selon les besoins et les ressources des localités ». Le ministère prend dans les années 1830 quelques mesures destinées à favoriser l'enseignement du dessin linéaire dans les écoles élémentaires, voire celui de la géométrie proprement dite. Quant aux écoles de filles, elles ne sont pas concernées par la loi Guizot. Il faut attendre une ordonnance royale de 1836 pour que la division en deux degrés soit étendue à l'enseignement féminin. Celui-ci doit comprendre "les éléments du dessin linéaire ", tant au degré élémentaire qu'au degré supérieur, mais l'étude de la géométrie n'est, en revanche, pas prévue. Le dessin linéaire représente donc, dans les 
établissements féminins comme dans les écoles élémentaires de garçons, le principal moyen d'initier les élèves à celle-ci.

La loi Falloux du 15 mars 1850 marque un coup d'arrêt, voire un retour en arrière. Elle fait perdre à l'enseignement primaire supérieur son existence légale (en réalité, il ne disparaît pas totalement) et le nouveau programme, qui concerne les écoles primaires de filles comme celles de garçons, est resserré autour des matières fondamentales : français et calcul. La géométrie est exclue: seules sont conservées, mais seulement comme matières facultatives, ses applications «utiles» - dessin linéaire, arpentage et nivellement. Cette suppression de la géométrie proprement dite est loin d'être politiquement neutre. Pour le nouveau pouvoir conservateur, l'extension donnée à l'instruction primaire sous la monarchie de Juillet représente une menace pour le bon ordre social. D'une certaine façon en effet, l'accession des élèves primaires à des connaissances traditionnellement réservées à l'enseignement secondaire - ainsi la géométrie - peut mettre en péril le cloisonnement entre l'école du peuple et celle des élites. À la fin du Second Empire, le ministre Victor Duruy permet de revenir partiellement à la situation antérieure: l'article 9 de la loi du 21 juin 1865 sur l'enseignement secondaire spécial inscrit de nouvelles matières facultatives au programme de l'enseignement primaire, parmi lesquelles «les éléments de la géométrie ».

Cette limitation des programmes sous le Second Empire se fait toutefois au profit d'une pédagogie plus concrète, davantage ancrée dans le réel. Plus, peut-être, que les matières facultatives, comme le dessin linéaire ou l'arpentage (encouragé par le ministre Fortoul en 1854), c'est l'enseignement du système métrique qui permet alors de dispenser quelques notions de géométrie aux élèves primaires. Ainsi, dans un article publié en 1855 dans une revue pédagogique proche du ministère, l'inspecteur primaire Jean-Jacques Rapet recommande aux instituteurs non seulement d'exercer leurs élèves « à mesurer toute espèce de quantités, des longueurs, des surfaces et des volumes ", mais aussi de donner "à propos du système métrique, toutes les notions de géométrie qui en sont le complément indispensable, et toutes celles qui sont réellement utiles, sinon nécessaires à tout le monde "`. Une décennie après l'adoption définitive du système métrique décimal et l'abrogation des anciens poids et mesures, il s'agit d'expliquer celui-ci, d'en montrer la logique, d'en faire sentir l'utilité quotidienne. À la fin des années 1860 , les écoles primaires parisiennes offrent un exemple d'une telle conception: l'étude des principales figures géométriques y est effectuée (au cours supérieur, c'est-à-dire en fin de cursus) dans le cadre de l'«application du système métrique à la mesure des surfaces et des volumes ${ }^{10}$.

\section{Une leçon de choses géométriques ?}

11 C'est la loi sur l'instruction primaire du 28 mars 1882 qui permet, après l'arrivée au pouvoir des républicains, de rompre avec les restrictions imposées par la loi Falloux. En particulier, la distinction entre matières obligatoires et facultatives est supprimée. Au calcul et au système métrique, qui formaient la base de l'enseignement mathématique primaire sous le Second Empire, sont substitués les «éléments des sciences mathématiques ». Un tel changement de vocabulaire est hautement symbolique: il indique la volonté de diversifier les connaissances enseignées dans le primaire, mais aussi de les rehausser. Commun aux écoles de filles et aux écoles de garçons ${ }^{11}$, le nouveau plan d'études des écoles primaires (arrêté du 27 juillet 1882) prévoit, outre le calcul et 
l'arithmétique, un enseignement de géométrie à tous les niveaux et non en fin de cursus seulement, comme c'était généralement le cas auparavant.

Deux facteurs contribuent au renouvellement de l'enseignement mathématique à l'école primaire, et notamment celui de la géométrie: l'organisation pédagogique et les méthodes d'enseignement. En premier lieu, le nouveau plan d'études adopte le principe de l'enseignement concentrique : l'enseignement est divisé en trois cours - élémentaire, moyen, supérieur - basés sur le même programme, de telle sorte que les élèves revoient chaque année les connaissances acquises antérieurement tout en les approfondissant. Non seulement il est alors logique que la géométrie soit enseignée dès l'entrée à l'école, mais cette organisation des études permet aux élèves d'aborder presque aussitôt la géométrie dans l'espace, contrairement à la tradition de la géométrie « classique » qui fait se succéder le plan et l'espace.

En second lieu, la «rénovation pédagogique » prônée par le ministère de l'Instruction publique incite à une révision des méthodes. Fondé sur l'observation du réel, l'enseignement doit être "intuitif et pratique » et procéder « du connu à l'inconnu, du facile au difficile ». Le programme de géométrie de 1882 reprend le principe de la « leçon de choses ", mais est également inspiré des conceptions du pédagogue suisse Pestalozzi (1746-1827), conceptions revenues à la mode dans les premières décennies de la Troisième République ${ }^{12}$ : à l'observation des figures géométriques et à l'évaluation à vue des grandeurs (cours élémentaire), succèdent leur représentation graphique et l'étude de leurs propriétés (cours moyen), puis une approche plus théorique avec des «notions sommaires sur la géométrie plane» (cours supérieur). Observer, reconnaître, nommer, comparer, mesurer, dessiner : telles sont les activités auxquelles les élèves, surtout les plus jeunes, sont désormais invités à se livrer dans le cadre du cours de géométrie. La mesure des surfaces et des volumes n'est pas mise à l'écart pour autant, bien au contraire. Celle-ci demeure en effet un élément essentiel du programme d'arithmétique, en liaison avec l'étude du système métrique. Sans référence explicite à la géométrie, les épreuves du certificat d'études primaires (qui sanctionne les études élémentaires) témoignent de cette priorité donnée - et pour longtemps - au système métrique et aux questions de mesurage dans l'enseignement mathématique primaire ${ }^{13}$.

Plus que les instructions officielles qui concernent l'ensemble des matières, l'article "Géométrie » du Dictionnaire de pédagogie et d'instruction primaire de Ferdinand Buisson renseigne sur les méthodes et les objectifs poursuivis ${ }^{14}$. Son auteur - l'inspecteur général Paul Leyssenne - y prône un enseignement concret, utilisant des solides en bois, en terre ou en carton, pour les plus jeunes élèves tout du moins : "Cet enseignement ne doit être qu'une leçon de choses appliquée [...] à des objets de formes régulières et mesurables ». Mais le discours change de tonalité lorsqu'il s'agit du cours moyen ou du cours supérieur. À ce niveau, le cours de géométrie vaut surtout pour son utilité pratique: il doit faciliter "l'intelligence du système métrique» et permettre l'évaluation des surfaces et des volumes. On retrouve ainsi la priorité donnée au système métrique. En outre, Leyssenne fait clairement la distinction entre un enseignement primaire élémentaire privilégiant l'intuition sensible et l'activité des élèves - le dessin linéaire est mis à contribution - et un enseignement primaire supérieur où la géométrie, bien que dirigée vers les applications pratiques, repose sur des démonstrations rigoureuses ${ }^{15}$. C'est, dans une large mesure, la position qui est officiellement adoptée pour l'enseignement des sciences physiques et naturelles : selon l'arrêté du 27 juillet 1882, celles-ci doivent être "présentées d'abord sous la forme de leçons de choses, et plus tard étudiées méthodiquement ${ }^{16}$. Mais une 
telle position, qui clive les méthodes d'enseignement en fonction de l'âge des enfants, ne fait pas l'unanimité. Dans une conférence donnée à des instituteurs et des institutrices, François Vintéjoux - un professeur du secondaire - se prononce en faveur d'une initiation précoce et progressive au raisonnement mathématique afin d'habituer les élèves "à ne rien affirmer à la légère ", et recommande d'aborder quelques démonstrations de géométrie dès le cours moyen ${ }^{17}$. On le voit, dans les années 1880 , la question de la place respective de l'expérience et du raisonnement logique en géométrie n'est pas résolue.

On assiste d'ailleurs, au tournant du siècle, à une forte remise en cause de l'enseignement «classique » de la géométrie au profit d'un mode d'exposition privilégiant l'expérience concrète, qui s'appuie sur des activités de dessin - dessin linéaire ou dessin géométrique - afin d'éclairer mais aussi d'appliquer les notions étudiées. L'enjeu est autant épistémologique que pédagogique : cette remise en cause, qui intervient dans le cadre d'une réflexion globale sur l'enseignement mathématique au niveau moyen (écoles primaires supérieures ou premier cycle du secondaire), est suscitée par des mathématiciens, comme Émile Borel ou Jules Tannery, désireux d'affirmer le caractère expérimental de leur discipline, et se traduit notamment par un rejet de l'exposé euclidien classique, jugé trop abstrait. À la suite de la grande réforme de l'enseignement secondaire de 1902, elle va conduire, en 1905, à une importante refonte des programmes de mathématiques du premier cycle $B$ (sans latin) ${ }^{18}$, lesquels seront repris en partie dans l'enseignement primaire supérieur en 1909.

16 Dans le primaire, au couple, désormais traditionnel, formé par la géométrie et le dessin, vient s'adjoindre une troisième composante: le travail manuel. Introduit dans les programmes de l'école primaire par la loi du 28 juin 1882, l'enseignement du travail manuel devait, à l'origine, permettre d'initier les élèves primaires au maniement des principaux outils pour le travail du bois et du fer. Mais dès les années 1890, les écoles élémentaires parisiennes s'orientent, sous l'impulsion de René Leblanc - un ancien instituteur devenu inspecteur général - vers un travail manuel "sans atelier", privilégiant le caractère éducatif plutôt que les finalités professionnelles. Essentiellement fondé sur des exercices pratiques tels que pliages ou réalisations de solides géométriques, celui-ci met en jeu les notions étudiées dans le cours de géométrie, et peut mener les élèves vers des activités de dessin ou de mesurage, voire des résolutions graphiques de problèmes géométriques simples ${ }^{19}$. En 1907, Leblanc propose ainsi une série d'exercices de " géométrie expérimentale » destinés à constater certaines propriétés géométriques et à donner une approche plus intuitive des notions théoriques ${ }^{20}$.

17 Le nouveau programme de première année des écoles primaires supérieures de 1909 intègre, au niveau national cette fois, cette nouvelle collaboration disciplinaire. Ainsi, il est recommandé aux maîtres « de relier entre eux les enseignements de la géométrie, du dessin et du travail manuel ». Si le cours de dessin conduit à des mesures ou des tracés précis et permet d'introduire la géométrie de l'espace et les projections, des «exercices de géométrie expérimentale» (découpage, cartonnage, coupe de plâtre) sont également prévus au programme de travail manuel : «Bien des vérités géométriques essentielles peuvent être mises en évidence au moyen d'exercices de (géométrie expérimentale > figurant au programme de travaux manuels: on ne manquera pas de les faire constater par les élèves; la démonstration rigoureuse des théorèmes qui les traduisent se trouvera ensuite fort simplifiée ». Auxiliaire pédagogique du cours de géométrie au même titre que le dessin, le travail manuel le deviendra également au niveau de l'école élémentaire en 1923. Ces interactions 
disciplinaires resteront largement opératoires dans l'enseignement primaire jusqu'à la réforme des mathématiques modernes des années 1970.

\section{En guise de conclusion}

Bien que l'étude de la géométrie ne soit pas une priorité de l'école primaire au XIX ${ }^{\mathrm{e}}$ siècle, son intégration progressive dans l'enseignement mathématique dispensé, d'abord au niveau primaire supérieur, ensuite au niveau élémentaire, impose d'accorder contenus et méthodes avec les finalités de cet ordre d'enseignement. Se démarquant de celle, plus abstraite et plus spéculative, enseignée dans les lycées ou les collèges du secondaire, la géométrie qui s'élabore ainsi privilégie le «faire » et l'expérience sensible en mobilisant le dessin puis le travail manuel. Cette coopération disciplinaire n'est pas sans conséquences: non seulement elle favorise le contact des plus jeunes élèves avec des objets et des concepts géométriques, mais elle remet en cause les modalités " classiques » de l'enseignement de la science d'Euclide. Elle témoigne ainsi de la capacité de l'école à déranger les cadres traditionnels de l'enseignement pour bâtir des formes didactiques originales.

\section{NOTES}

1. Exposé des motifs du projet de loi sur l'instruction primaire, présenté à la Chambre des députés par M. le ministre secrétaire d'État de l'Instruction publique, 2 janvier 1833, Bulletin universitaire, tome 3 , p. 249. On retrouvera les textes législatifs ou réglementaires mentionnés dans cet article dans D'ENFERT R.; L'enseignement mathématique à l'école primaire, de la Révolution à nos jours. Textes officiels. Tome 1:1791-1914. Paris, INRP, 2003 (avec la collaboration d'H. Gispert et J. Hélayel).

2. Sur l'enseignement secondaire des mathématiques au XIXe siècle, voir BELHOSTE B : Les sciences dans l'enseignement secondaire français. Textes officiels, 1789-1914. Paris, INRP Économica, 1995.

3. FRANCEEUR L.-B.: Le dessin linéaire d'après la méthode de l'enseignement mutuel. Paris, Colas, 1819. De nombreux rapports relatifs au dessin linéaire sont publiés dans le Journal d'éducation, organe de la Société pour l'instruction élémentaire créée en 1815 afin de promouvoir l'enseignement mutuel. Sur ce point, voir D'ENFERT R. : L'enseignement du dessin en France. Figure humaine et dessin géométrique (1750-1850). Paris, Belin, 2003.

4. LANCELOT : Dessin linéaire et géométrie pratique, suivi d'un tarif de réduction du bois carré et en grume, et de la concordance des calendriers grégoriens et républicains. Châlons, Impr. de Boniez-Lambert, 1827.

5. FRANCEEUR L.-B. : Dessin linéaire et arpentage pour toutes les écoles primaires. Paris, Colas, 1832, p. 7.

6. Le projet initial présenté par Guizot ( 2 janvier 1833 ) prévoyait de porter « le dessin linéaire, l'arpentage, et les autres applications de la géométrie pratique» au programme de l'enseignement primaire supérieur. 
7. AN/F/17/9824: «Projet d'organisation pour le collège du Havre présenté par le principal à MM. les membres du bureau d'administration le 29 janvier 1837, et lu par M. le Maire en conseil municipal le 16 février suivant ", 19 janvier 1837.

8. Voir par exemple SONNET H.: Premiers éléments de géométrie avec les principales applications au dessin linéaire, au lever des plans, à l'arpentage, etc. Paris, Hachette, 1845.

9. RAPET.J.-J. : « De la direction à donner par les instituteurs à leur enseignement ", in Bulletin de l'instruction primaire. Tome 3, $\mathrm{N}^{\circ} 5$, mars 1855 , p. 122.

10. «Instruction générale à $M M$. les inspecteurs primaires sur la mise à exécution du règlement d'organisation pédagogique du département de la Seine", in Organisation pédagogique. Programmes et instructions. Extrait du Bulletin de l'enseignement primaire du département de la Seine. Paris, Charles Mourgues Frères, 1870, pp. 21-58.

11. Ce plan d'études ne concerne en réalité que les écoles primaires élémentaires. Dans l'enseignement primaire supérieur, réactivé dès la fin des années 1870, l'enseignement féminin reste peu concerné par la géométrie avant 1893.

12. Voir notamment GUILLAUME J. : article «Pestalozzi », in BUISSON F. (dir.) : Dictionnaire de pédagogie et d'instruction primaire, 1re partie, tome 2. Paris, Hachette, 1887, pp. 2283-2358. Cet article est l'un des plus longs du Dictionnaire.

13. Jusqu'en 1917, l'épreuve écrite comprend « deux questions d'arithmétique portant sur les applications du calcul et du système métrique, avec solution raisonnée ». Elle ne sera guère modifiée par la suite. Lorsqu'en 1923, l'examen du certificat est scindé en deux parties, l'épreuve écrite de la seconde partie comporte "un problème d'arithmétique ou de géométrie ». Cette mesure sera abrogée l'année suivante.

14. LEYSSENNE P. : article "Géométrie », in BUISSON F. (dir.) : Dictionnaire de pédagogie et d'instruction primaire, op. cit., 1re partie, tome 1, pp. 1162-1166.

15. Ibid., L'enseignement des écoles normales primaires est également concerné.

16. Voir également BOUTAN A. : article "Expériences", in BUISSON F. (dir.) : Dictionnaire de pédagogie et d'instruction primaire, op. cit., 1re partie, tome 1, pp. 972-974. L'auteur distingue deux modèles pédagogiques en fonction de l'âge des enfants : descriptif pour les petits, explicatif pour les plus grands. Sur l'enseignement primaire des sciences physiques et naturelles au XIXe siècle, voir KAHN P. : La leçon de choses. Naissance de l'enseignement des sciences à l'école primaire. Villeneuve d'Ascq, Presses universitaires du Septentrion, 2002.

17. VINTÉJOUX F. : «L'enseignement de l'arithmétique et de la géométrie à l'école primaire », in Revue pédagogique, nouvelle série. Tome 10, N 3, 15 mars 1887, pp. 223-232.

18. Voir BELHOSTE B. : Les sciences dans l'enseignement secondaire, op. cit., pp. 55-61.

19. Sur ce point, voir la contribution de René Leblanc à l'article « Manuel (Travail)» du Nouveau dictionnaire de pédagogie et d'instruction primaire, Paris, Hachette, 1911, pp. 1214-1219. Sur l'évolution de l'enseignement du travail manuel au cours de la période et notamment les liens avec celui la géométrie, voir LEBEAUME J. : École, technique et Travail manuel, Nice, Z'éditions, 1995 ; ainsi que D'ENFERT R. : « i Manuel (Travail) 〉 : préparer au métier ou éduquer », in DENIS D. et KAHN P. (dir.): L'école républicaine et la question des savoirs. Enquête au cœur du Dictionnaire de pédagogie de Ferdinand Buisson, Paris, Éditions du CNRS, pp. 199-222.

20. LEBLANC R. : La réforme des écoles primaires supérieures. Paris, Hachette, 1907, pp. 75-86. 


\section{RÉSUMÉS}

Introduite officiellement par la loi Guizot de 1833, la géométrie enseignée à l'école primaire au XIXe siècle doit privilégier les applications pratiques et se démarquer ainsi de celle, plus théorique, enseignée dans le secondaire. Se constitue alors une géométrie bien spécifique, qui mobilise largement les activités graphiques. On retrace ici cette élaboration disciplinaire et on en montre les différents enjeux, pédagogiques, épistémologiques, voire idéologiques.

Officially introduced by the Guizot law of 1833, geometry as it was taught in the primary schools in the XIXth century, must focus upon practical applications, thereby differentiating itself from the theoretical geometry taught in the secondary schools. As a result, a particular type of geometry is developed, largely calling upon draughtsmanship skills. This article unfolds the development of this discipline

\section{INDEX}

Mots-clés : enseignement primaire, géométrie, histoire, XIXe siècle

Keywords : geometry, history, primary school education, XIXth century

\section{AUTEUR}

\section{RENAUD D'ENFERT}

Service d'Histoire de l'Education de l'INRP, IUFM de Versailles 